\section{Kompressionsstrumpf für trockene Haut}

Der VenoTrain micro balance verbindet den hohen Tragekomfort des VenoTrain micro mit einer spezifischen Pflegeemulsion, die den transepidermalen Wasserverlus reduziert. Damit ermöglicht der medizinische Kompressionsstrumpf eine optimale Therapie für Patienten mit empfindlicher und trockener Haut. Der Kompressionsstrumpf ist ab sofort mit $100 \mathrm{ml}$ Reload-Emulsion und einem Zerstäuberaufsatz im Fachhandel erhältlich.

Nach Informationen von Bauerfeind

\section{Salbe statt Emulsion}

Der Wirkstoff helles Ammoniumbituminosulfonat ist ab sofort nicht mehr auf dem Weltmarkt verfügbar. Demzufolge können Arzneimittel wie Aknemycin ${ }^{\circledR}$ Emulsion nicht mehr hergestellt werden. Ein geeignetes Nachfolgepräparat ist die Aknemycin ${ }^{\circledR}$ Salbe von Almirall Hermal: Aknemycin ${ }^{\circledR}$ Salbe ist ein Monotherapeutikum und enthält 2\% Erythromycin. Die galenischen Grundlagen der Aknemycin ${ }^{\circledR}$ Salbe und Emulsion sind trotz der unterschiedlichen Bezeichnung nahezu identisch.

Nach Informationen von Almirall Hermal

\section{Compliance bei Psoriasis erhöhen}

Trotz des hohen Leidensdrucks ist die Compliance in der Psoriasis-Therapie erschreckend gering: Bis zu 40\% der Patienten führen ihre Behandlung nicht wie verordnet aus. Die bewährte Fixkombination von Calcipotriol mit Betamethason in innovativer Lipo-Gel-Galenik, die seit Juni 2010 erhältlich ist, hilft die Compliance zu erhöhen: Daivobet ${ }^{\circledR} \mathrm{Gel}$ für den Körper ist farblos, transparent, duftneutral und verzichtet bewusst auf austrocknenden Alkohol. Das Lipo-Gel lässt sich leicht verteilen, zieht schnell ein und besitzt mit einmal täglichem Auftragen ein patientenfreundliches Therapieregime.

Nach Informationen von LEO Pharma

\title{
Optische Biopsie in Echtzeit
}

\begin{abstract}
- Mit der konfokalen Laserscanmikroskopie lassen sich Hautveränderungen wie Sonnenschäden, Präkanzerosen und melanozytäre sowie nicht-melanozytäre Hauttumoren in verschiedenen Stadien nicht-invasiv, schnell und präzise analysieren. Damit ermöglicht die innovative, patientenfreundliche Untersuchungsmethode eine frühzeitige Diagnose und Therapie sowie eine beliebig lange Kontrolle geschädigter Hautareale.

In mehreren Studien wurden beispielsweise Diagnosekriterien zur Erkennung von aktinischen Keratosen und Basalzellkarzinomen entwickelt [Expert Rev Dermatol 2008; 3: 557-67; J Am Acad Dermatol 2004; 51: $923-30]$.
\end{abstract}

Darüber hinaus gestattet ein DiagnoseAlgorithmus die Differenzierung zwischen Lentigo maligna und benignen Hautflecken [J Invest Dermatol advance online publica- tion, 15. April 2010; DOI:10.1038/jid.2010.84]. Auch eine aktuelle Übersichtsarbeit bestätigt die Eignung der konfokalen Laserscanmikroskopie für die Diagnostik melanozytärer und nicht-melanozytärer Hauttumoren [online on Wiley InterScience; DOI:10.1111/j.16100387.2009.07333.x].

Den Anwendern von VivaScope-Geräten bietet deren Hersteller ein kostenloses und umfassendes Trainingsprogramm an, das sich aus aufeinander aufbauenden Modulen zusammensetzt. Außerdem gibt es ein Expertentraining für Fortgeschrittene an zwei Kompetenzzentren mit den Schwerpunkten melanozytäre (Universität Modena) bzw. nicht-melanozytäre (Berliner Charité) Läsionen und ein Online-Readers-Training. Weitere Infos: www.vivascope.eu.

Nach Informationen von Mavig

\section{Pedikulozid mit hoher Erfolgsquote}

_ Ein Problem bei der Bekämpfung der Kopflaus (Pediculus humanus capitis) sind zunehmende Resistenzen der Parasiten. So wies Prof. Jörg Heukelbach aus Fortaleza, Brasilien, darauf hin, dass in einigen Industrie- und Schwellenländern praktisch alle Kopfläuse unempfindlich gegen Permethrin sind. „Bei Dimeticon, einem Silikonöl-Gemisch, sind Resistenzen dagegen kaum möglich“, sagte Heukelbach. „Es wirkt rein physikalisch. "Gute Erfahrungen gebe es mit dem Zwei-Stufen-Dimeticon $\mathrm{NYDA}^{\circledR}$ : „Es beinhaltet ein niedermolekulares Dimeticon mit sehr geringer Oberflächenspannung, das in die Stigmen der Läuse und auch in die Aeropylen der Eier hineinkriecht“, erklärte der Epidemiologe. Die Parasiten ersticken danach.

Heukelbach präsentierte eine eigene Studie an 145 brasilianischen Kindern mit starkem Kopflausbefall. Sie besuchten gemeinsam ein Ferienlager und wurden am ersten und achten Tag verblindet mit dem Zwei-Stufen-Dimeticon- oder einem PermethrinPräparat behandelt. „Am Tag neun waren $97,2 \%$ der mit NYDA $^{\circledR}$ therapierten Kinder frei von lebensfähigen Läusen“, berichtete Heukelbach.,,Mit Permethrin waren es 67,6\%." Außerdem war die kosmetische Akzeptanz des Silicongemischs signifikant höher.

rdf

Presseworkshop „Dimeticone in der Kopflaustherapie - sicher und effektiv", Hamburg, 20. Mai 2010 in Hamburg (Veranstalter: Pohl-Boskamp)

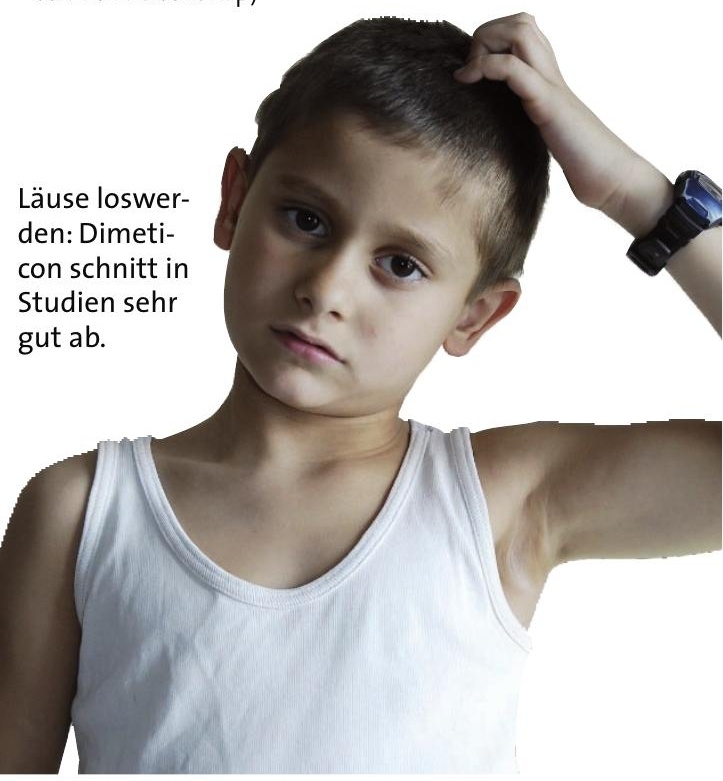

